\title{
Smalltalk Graphics as a Visual Aid to Acquiring Query Language Skills over a Semantic Data Model
}

\author{
Nicholas Cole and Peter M.D. Gray \\ Department of Computing Science \\ University of Aberdeen \\ Aberdeen, Scotland AB24 3UE \\ E-Mail:pgray@csd.abdn.ac.uk
}

\begin{abstract}
We present an approach to visualisation using Smalltalk graphics on a Silicon Graphics machine. This is compared with the previous approach (using $\mathrm{C}$ and 3-D graphics) to capturing the query by graphic input. We are investigating a way to link textual and graphic modes of query formation, which enables us to make good use of Smalltalk graphics. We show the need to use the full Model-View-Controller paradigm. We believe the resulting system helps the user to grasp the style and clause structure of the Daplex language, by relating visual cues to linguistic ones. Words in the generated Daplex are mouse active and cross-reference to schema objects in the graphics window This approach would generalise to other semantic data models, as used by ODMG.
\end{abstract}

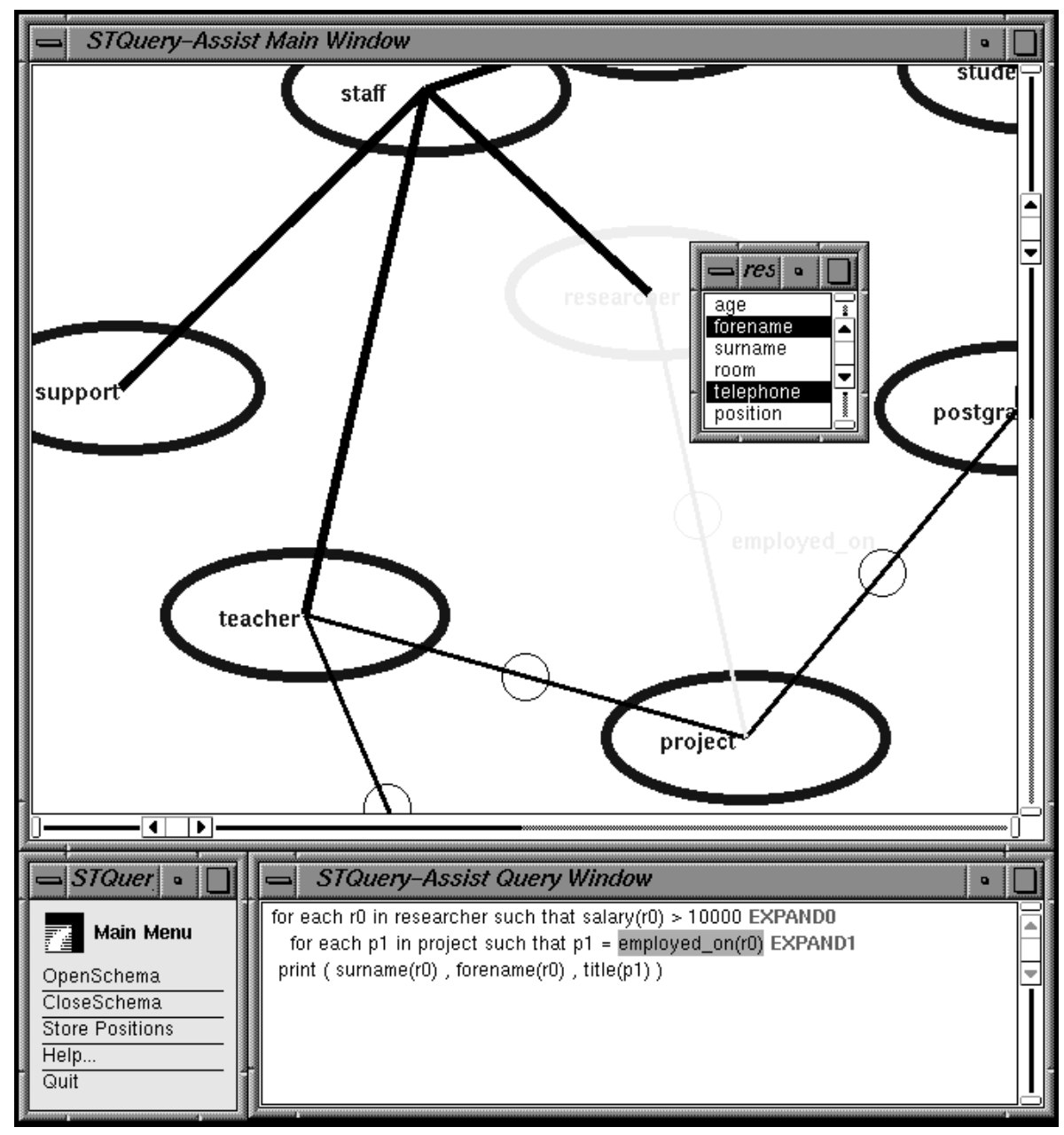

Figure 1: An Example of the Above-Described Application

Interfaces to Databases (IDS-3) 